\title{
Single Top Production at HERA and THERA
}

\author{
A.T.ALAN and A.SENOL \\ Department of Physics, Faculty of Sciences and Arts, \\ Abant Izzet Baysal University,14280 Gölköy, Bolu, TURKEY
}

(Dated: October 26, 2018)

\begin{abstract}
We study the single top production mediated by flavor changing neutral current via both of the $t-q-\gamma$ and $t-q-Z$ vertices (here q represents $\mathrm{c}$ and $\mathrm{u}$ quarks) in $e p$ collisions at two colliders HERA and THERA. Contribution of the second vertex becomes even more important as the couplings take more improved values provided by the higher luminosities of colliders. In addition to these improvements if the CM energy of the collider is increased, the production will be dominated by the anomalous $t-q-Z$ vertex.
\end{abstract}




\section{INTRODUCTION}

It is well known that within the Standard Model (SM), single top quark production is negligible because of the flavor conservation in the leading order.However in a wide class of dynamical models extending the SM this possibility exists due to the large top mass close to the electroweak symmetry breaking scale. Flavor Changing Neutral Current (FCNC) interactions of top quarks has a particular importance. In these models the top quarks is predicted to have significantly large FCNC interactions [1, 2]. Production of single top quarks via FCNC vertices was extensively studied at $e^{+} e^{-}$[3, 团] and at hadron colliders [5, 6, 7] . At HERA, as a lepton-hadron type collider, the same production was first investigated in [8]. In this reference only the charm distribution in the proton is considered. The H1 Collaboration searched for the production by considering both of anomalous vertices $t-u-\gamma$

and $t-c-\gamma$ from the fact that HERA has much higher sensitivity to $\kappa_{u \gamma}$ than to $\kappa_{c \gamma}$, due to more favorable parton density 10 .

In this paper we analyze anomalous single top production considering both of the $\kappa_{q \gamma}$ and $\kappa_{q Z}$ couplings to see especially the contribution of the second coupling. In order to make illustration clear we present the numerical results for HERA and THERA [9] colliders with the CM energies $\sqrt{S}=320$ and $1000 \mathrm{GeV}$ respectively.

\section{ANOMALOUS TOP DECAYS}

We consider the most general effective Lagrangian proposed in [3] (generalized by the H1 Collaboration to include $u \rightarrow t$ transition [14]) to describe the anomalous top decays $t \rightarrow q \gamma$ and $t \rightarrow q Z$ :

$$
\mathcal{L}_{e f f}=\sum_{U=u, c} i \frac{e e_{U}}{\Lambda} \bar{t} \sigma_{\mu \nu} q^{\nu} \kappa_{\gamma, U} U A^{\mu}+\frac{g}{2 \cos \theta_{W}} \bar{t}\left[\gamma_{\mu}\left(v_{Z, U}-a_{Z, U} \gamma^{5}\right)+i \frac{1}{\Lambda} \sigma_{\mu, \nu} q^{\nu} \kappa_{Z, U}\right] U Z^{\mu}+h . c .
$$

where $\sigma_{\mu, \nu}=(i / 2)\left[\gamma^{\mu}, \gamma^{\nu}\right], \theta_{W}$ is the Weinberg angle, $e_{U}$ is the electric charge of up-type quarks, and $\Lambda$ denotes the scale up to which the effective theory is assumed to hold. By convention we set $\Lambda=m_{t}$ in the following.

We find:

$$
\Gamma(t \rightarrow q \gamma)=\alpha e_{q}^{2} \kappa_{q \gamma}^{2} m_{t}\left(1-\frac{m_{q}^{2}}{m_{t}^{2}}\right)^{3}
$$




$$
\Gamma(t \rightarrow q Z)=\frac{\alpha m_{t}}{4 \sin ^{2} \theta_{W} \cos ^{2} \theta_{W}}\left(1-\frac{M_{Z}^{2}}{m_{t}^{2}}\right)^{2}\left[\kappa_{q z}^{2}\left(1+\frac{M_{Z}^{2}}{2 m_{t}^{2}}\right)-3 v_{q} \kappa_{q z}+\left(a_{q}^{2}+v_{q}^{2}\right)\left(1+\frac{m_{t}^{2}}{M_{Z}^{2}}\right)\right]
$$

where $\alpha$ is the fine structure constant, $M_{Z}$ is the mass of $Z$ boson. Using the standard decay value $\Gamma(t \rightarrow q W)=1.397 \mathrm{GeV}$ and the experimental constrains [11]

$$
\begin{aligned}
& B R(t \rightarrow q \gamma)<3.2 \% \quad C L 95 \% \\
& B R(t \rightarrow q Z)<33 \% \quad C L 95 \%
\end{aligned}
$$

the upper limits on the couplings are easily obtained;

$$
\kappa_{q \gamma}<0.24 \quad \kappa_{q Z}<0.27
$$

In obtaining these values we assumed $\operatorname{Im} \kappa_{q Z}=0$ and since $m_{q} \ll m_{t}$, we neglected the light quark masses.

\section{THE PRODUCTION OF SINGLE TOP QUARKS AT HERA AND THERA}

Differential production cross sections corresponding to the diagrams mediated by $\gamma, Z$ and their interference term respectively are;

$$
\begin{aligned}
\frac{d \hat{\sigma}_{\gamma}}{d \hat{t}}= & \frac{e_{0}^{2} e^{4} \kappa_{q \gamma}^{2}}{8 \pi \hat{s}^{2} \hat{t}}\left[(2 \hat{s}+\hat{t})-\frac{2 \hat{s}(\hat{s}+\hat{t})}{m_{t}^{2}}-m_{t}^{2}\right] \\
\frac{d \hat{\sigma}_{Z}}{d \hat{t}=} & \frac{g_{Z}^{4}}{128 \pi \hat{s}^{2} m_{t}^{2}\left[\left(\hat{t}-M_{Z}^{2}\right)^{2}+\Gamma_{Z}^{2} M_{Z}^{2}\right]} \\
& \left\{\left(a_{e}^{2}+v_{e}^{2}\right) \kappa_{q Z}^{2}\left[(2 \hat{s}+\hat{t}) m_{t}^{2}-2 \hat{s}(\hat{s}+\hat{t})-m_{t}^{4}\right] \hat{t}\right. \\
& +2 \kappa_{q Z} m_{t}^{2}\left[v_{q}\left(a_{e}^{2}+v_{e}^{2}\right)\left(\hat{t}-m_{t}^{2}\right)-2 a_{e} v_{e} a_{q}\left(2 \hat{s}+\hat{t}-m_{t}^{2}\right)\right] \hat{t} \\
& \left.+\left[\left(a_{q}^{2}+v_{q}^{2}\right)\left(a_{e}^{2}+v_{e}^{2}\right)\left[2 \hat{s}^{2}+2 \hat{s} \hat{t}-(2 \hat{s}+\hat{t}) m_{t}^{2}+\hat{t}^{2}\right]-4 a_{e} v_{e} a_{q} v_{q}\left(2 \hat{s}+\hat{t}-m_{t}^{2}\right)\right] m_{t}^{2}\right\} \\
\frac{d \hat{\sigma}_{i n t}}{d \hat{t}}= & \frac{g_{Z}^{2} e_{0} e^{2} \kappa_{q \gamma}\left(\hat{t}-M_{Z}^{2}\right)}{16 \pi \hat{s}^{2} m_{t}^{2}\left[\left(t-M_{Z}^{2}\right)^{2}+\Gamma_{Z}^{2} M_{Z}^{2}\right]} \\
& \left\{a_{e} a_{q}\left(2 \hat{s}+\hat{t}-m_{t}^{2}\right) m_{t}^{2}+v_{e}\left[2 \hat{s}^{2} \kappa_{q Z}-\left(\hat{t}-m_{t}^{2}\right)\left(m_{t}^{2}\left(v_{q}+\kappa_{q Z}\right)-2 \hat{s} \kappa_{q Z}\right)\right]\right\}
\end{aligned}
$$

The total cross section is obtained by the integral of the sum of these three :

$$
\sigma_{\text {tot }}=\int_{x_{\text {min }}}^{1} f_{q}(x) d x \int_{t_{-}}^{t_{+}} \frac{d \hat{\sigma}}{d \hat{t}} d \hat{t}
$$


where $t_{-}=-\left(\hat{s}-m_{t}^{2}\right), t_{+}=t_{c u t}=-0.001 G e V$ and $x_{\text {min }}=m_{t}^{2} / S$.

$f_{q}(x)$ is the quark distribution function inside the proton [12, 13]:

$$
\begin{aligned}
f_{u}(x) & =A_{u} x^{\eta_{1}-1}(1-x)^{\eta_{2}}\left(1+\epsilon_{u} \sqrt{x}+\gamma_{u} x\right) \\
f_{c}(x) & =\frac{1}{2} N_{5} x^{2}\left[\frac{1}{3}(1-x)\left(1+10 x+4 x^{2}\right)+2 x(1+x) \ln x\right](n=2)
\end{aligned}
$$

where $A_{u}=0.8884, \eta_{1}=0.4710, \eta_{2}=3.404, \epsilon_{u}=1.628, \gamma_{u}=9.628$ and $N_{5}=36$.

Figure 1 13 display the photonic and total cross-section due to up quark distribution as a function of the center of mass energy (from HERA to THERA energy), taking, $\kappa_{u \gamma}=0.3$, 0.2, 0.1 (solid lines) and $\kappa_{u Z}=\kappa_{u \gamma}=0.3,0.2,0.1$ (dashed lines) respectively. These values for the coupling constants were chosen for purposes of demonstration only. Since the contribution of the charm quark distribution to the production cross sections is much less than that of the up distribution, instead of displaying the corresponding figures, we tabulated the cross-section values in Table 파 [II. Table $\mathbb{\|}$ is formed by considering only the photonic channel $\left(\kappa_{c \gamma}=0.1,0.2\right.$ and 0.3$)$. In forming Table [I and [II we considered both of the couplings $\kappa_{c \gamma}$ and $\kappa_{c Z}$ with the values as indicated and therefore tabulated the total cross section values at HERA and THERA colliders respectively.

\section{CONCLUSIONS}

In most of the single top searches the dominant contribution to the production is expected from the anomalous coupling $\kappa_{q \gamma}$ only. However the contribution due to $\kappa_{q Z}$ becomes important for the production with more stringent bounds on these couplings. From Figure 가 3 it is clear that contribution of the anomalous production vertex $t-q-Z$ is negligible only at HERA energies for $\kappa_{u V}=0.3$ (here $V$ refers to $\gamma$ and $Z$ ). On the other hand the

contribution of this vertex is increased in two ways; one is by increasing the center of mass energy and the other is as the couplings take more stringent values. Figure 3 shows that if they take the same value of 0.1 , the production is dominated by the anomalous vertex $t-q-Z$ at THERA energies. 


\section{Acknowledgments}

We would like to thank T.Tait for helpful discussion. This work was supported by Abant Izzet Baysal University Research Fund.

[1] H. Fritzsh, Phys.Lett. B224 (1989) 423.

[2] T. Han, R.D. Peccei, X. Zhang, Nucl.Phys. B434 (1995) 527.

[3] T.Han and J.L. Hewett, Phys.Rev. D60 (1999) 074015.

[4] V.F. Obraztsov S.R. Slabospitsky O.P. Yushchenko, Phys.Lett. B426 (1998) 393.

[5] T.Tait, C.P.Yuan, Phys.Rev. D63 (2001) 014018.

[6] T.Tait, C.P.Yuan, Phys.Rev. D55 (1997) 7300.

[7] E.Malkawi, T.Tait, Phys.Rev. D54 (1996) 5758.

[8] H. Fritzsch, D. Holtmannspoetter, Phys.Lett. B457 (1999) 186.

[9] H. Abramowicz et al. [TESLA-N Study Group Collaboration], TESLA: The superconducting electron positron linear collider with an integrated X-ray laser laboratory. Technical design report. Pt. 6: Appendices. Chapter 2: THERA: Electron proton scattering at $\mathrm{s}^{* *}(1 / 2)$ approx. 1-TeV," DESY-01-011.

[10] H1 Collaboration "Search for Single Top Production in $e^{ \pm} p$ collisions at HERA", contributed paper 516, LP2001, Rome.

[11] D.E. Groom et al., The Europen Physical Journal C15 (2000) 1.

[12] A. D. Martin, R. G. Roberts, W. J. Stirling, R. S. Thorne, Eur.Phys.J. C4 (1998) 463.

[13] J.F. Gunion and R.Vogt, hep-ph/9706252.

[14] This possibility is stated also in ref. [5] 


\begin{tabular}{|c||c|c|c|}
\hline$\kappa_{c \gamma}$ & 0.1 & 0.2 & 0.3 \\
\hline \hline$\sqrt{S}=320$ & $1.6 .10^{-3}$ & $6.2 .10^{-3}$ & $14.0 .10^{-3}$ \\
\hline$\sqrt{S}=1000$ & $0.7 .10^{-2}$ & $2.7 .10^{-2}$ & $6.2 .10^{-2}$ \\
\hline
\end{tabular}

TABLE I: Single top production cross section in pb due to charm quark distribution mediated by photon

\begin{tabular}{|c||l|l|l|l|}
\hline & $\kappa_{q Z}=0$ & $\kappa_{c Z}=0.1$ & $\kappa_{c Z}=0.2$ & $\kappa_{c Z}=0.3$ \\
\hline \hline$\kappa_{c \gamma}=0$ & $3.0 .10^{-3}$ & $3.2 .10^{-3}$ & $3.4 .10^{-3}$ & $3.7 .10^{-3}$ \\
\hline$\kappa_{c \gamma}=0.1$ & $5.3 .10^{-3}$ & $5.5 .10^{-3}$ & $5.7 .10^{-3}$ & $6.0 .10^{-3}$ \\
\hline$\kappa_{c \gamma}=0.2$ & $10.6 .10^{-3}$ & $10.8 .10^{-3}$ & $11.0 .10^{-3}$ & $11.3 .10^{-3}$ \\
\hline$\kappa_{c \gamma}=0.3$ & $19.0 .10^{-3}$ & $19.2 .10^{-3}$ & $19.5 .10^{-3}$ & $19.8 .10^{-3}$ \\
\hline
\end{tabular}

TABLE II: The total single top production cross section in pb due to charm quark distribution at HERA

\begin{tabular}{|c||c|c|c|c|}
\hline & $\kappa_{c Z}=0$ & $\kappa_{c Z}=0.1$ & $\kappa_{c Z}=0.2$ & $\kappa_{c Z}=0.3$ \\
\hline \hline$\kappa_{c \gamma}=0$ & $4.1 .10^{-2}$ & $4.2 .10^{-2}$ & $4.5 .10^{-2}$ & $4.9 .10^{-2}$ \\
\hline$\kappa_{c \gamma}=0.1$ & $5.0 .10^{-2}$ & $5.1 .10^{-2}$ & $5.4 .10^{-2}$ & $5.8 .10^{-2}$ \\
\hline$\kappa_{c \gamma}=0.2$ & $7.2 .10^{-2}$ & $7.3 .10^{-2}$ & $7.6 .10^{-2}$ & $8.1 .10^{-2}$ \\
\hline$\kappa_{c \gamma}=0.3$ & $10.7 .10^{-2}$ & $10.9 .10^{-2}$ & $11.2 .10^{-2}$ & $11.7 .10^{-2}$ \\
\hline
\end{tabular}

TABLE III: The total single top production cross section in pb due to charm quark distribution at THERA 


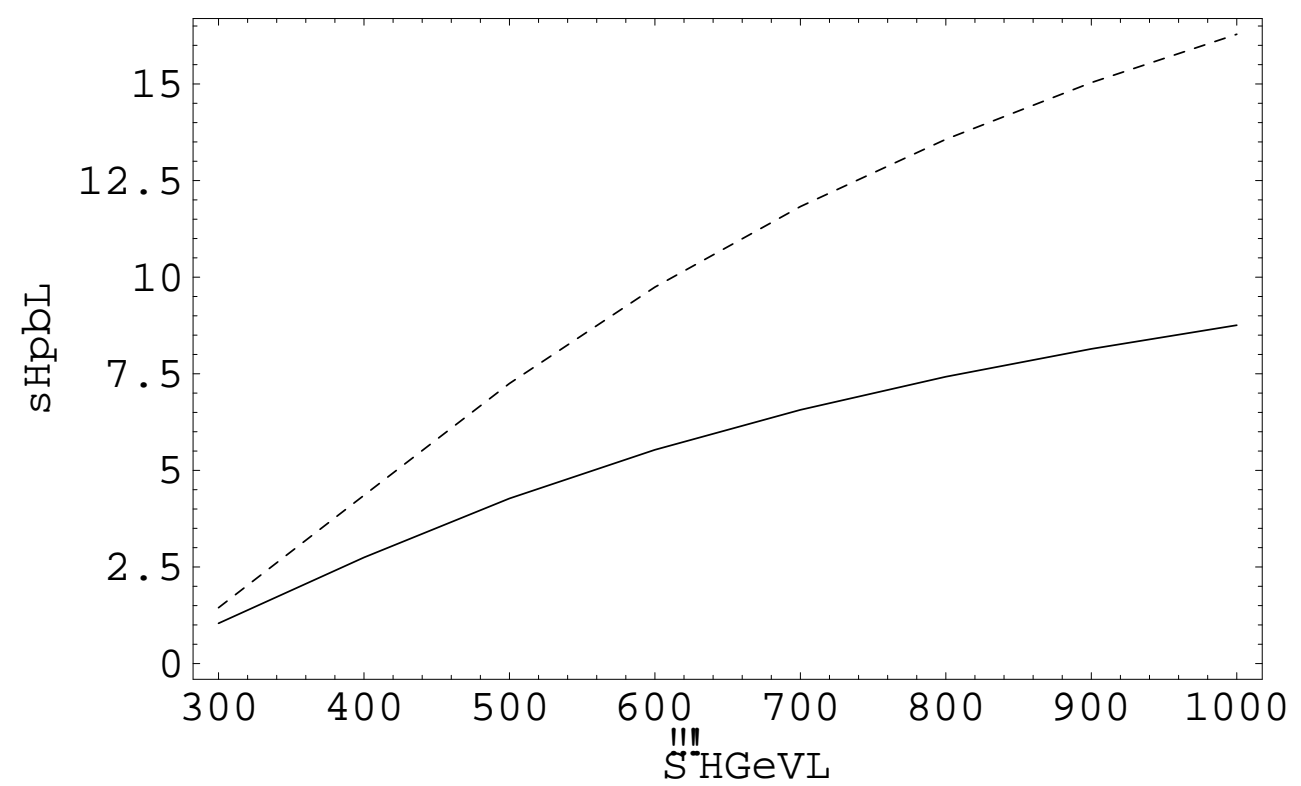

FIG. 1: Photonic (solid line) and total (dashed line) production cross section for the FCNC single top quark as a function of center of mass energy with $\kappa_{u \gamma}=0.3$ and $\kappa_{u \gamma}=\kappa_{u Z}=0.3$ respectively.

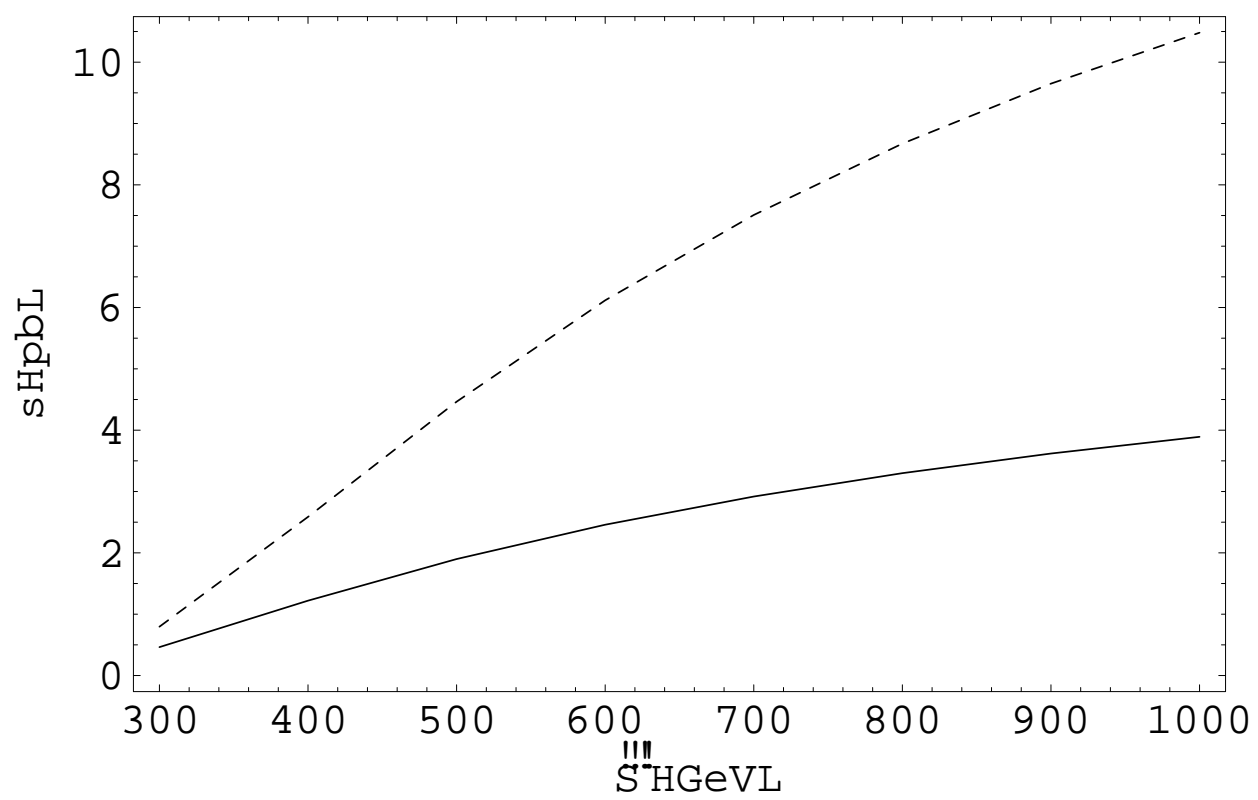

FIG. 2: Photonic (solid line) and total (dashed line) production cross section for the FCNC single top quark as a function of center of mass energy with $\kappa_{u \gamma}=0.2$ and $\kappa_{u \gamma}=\kappa_{u Z}=0.2$ respectively. 


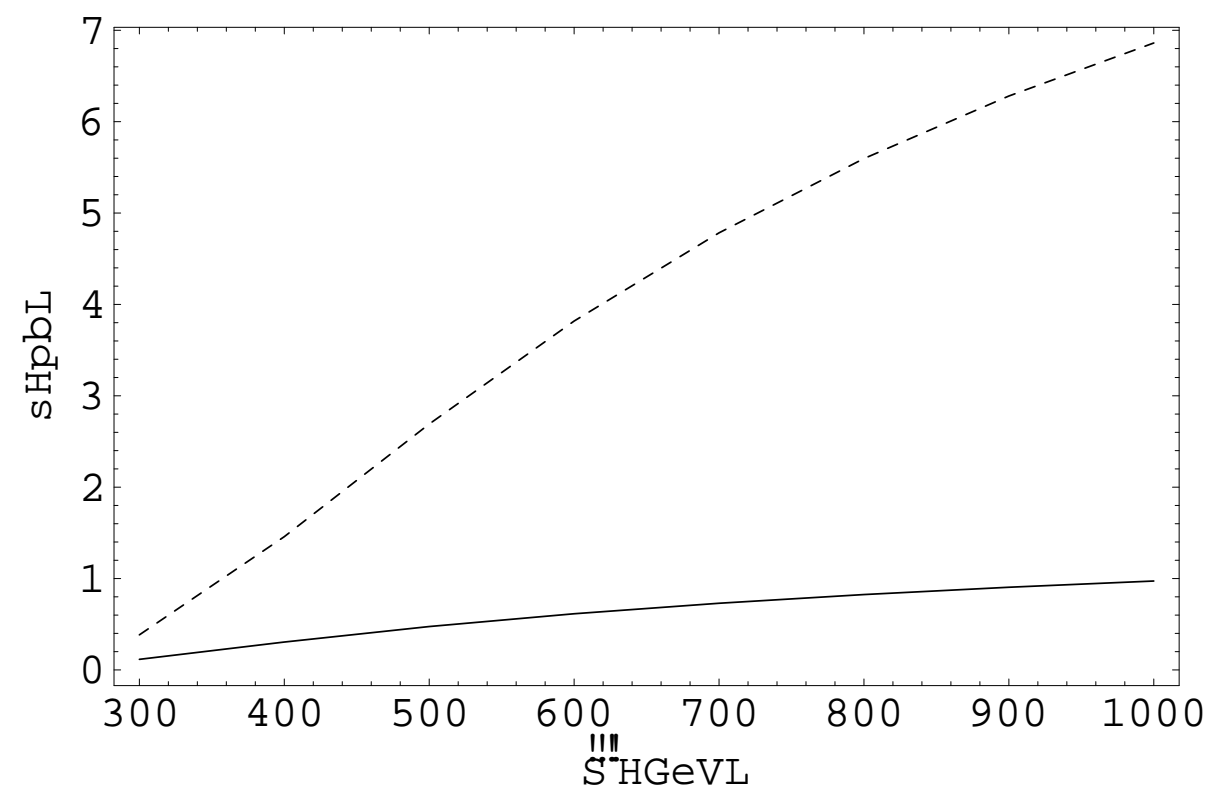

FIG. 3: Photonic (solid line) and total (dashed line) production cross section for the FCNC single top quark as a function of center of mass energy with $\kappa_{u \gamma}=0.1$ and $\kappa_{u \gamma}=\kappa_{u Z}=0.1$ respectively. 